\title{
Influence of Electric Field Coupling Model on the Simulated Performances of a GaN Based Planar Nanodevice
}

\author{
K. Y. Xu, ${ }^{1}$ Z. N. Wang, ${ }^{1}$ Y. N. Wang, ${ }^{1}$ J. W. Xiong, ${ }^{1}$ and G. Wang ${ }^{2}$ \\ ${ }^{1}$ Laboratory of Quantum Engineering and Quantum Materials, School of Physics and Telecommunication Engineering, \\ South China Normal University, Guangzhou 510006, China \\ ${ }^{2}$ State Key Laboratory of Optoelectronic Materials and Technologies, Sun Yat-sen University, Guangzhou 510275, China \\ Correspondence should be addressed to K. Y. Xu; xuky@scnu.edu.cn
}

Received 18 October 2013; Revised 18 December 2013; Accepted 19 December 2013

Academic Editor: Razali Ismail

Copyright (C) 2013 K. Y. Xu et al. This is an open access article distributed under the Creative Commons Attribution License, which permits unrestricted use, distribution, and reproduction in any medium, provided the original work is properly cited.

\begin{abstract}
The performances of a two-dimensional electron gas (2DEG) based planar nanodevice are studied by a two-dimensional-threedimensional (2D-3D) combined model and an entirely 2D model. In both models, 2DEGs are depicted by 2D ensemble Monte Carlo (EMC) method. However electric field distributions in the devices are obtained by self-consistently solving 2D and 3D Poisson equations for the 2D model and the 2D-3D model, respectively. Simulation results obtained by both models are almost the same at low bias while showing distinguished differences at high bias. The 2D model predicts larger output current and slightly higher threshold voltage of Gunn oscillations. Although the fundamental frequencies of current oscillations obtained by both models are similar, the deviation of wave shape from sinusoidal waveform obtained by the $2 \mathrm{D}$ model is more serious than that obtained by 2D-3D model. Moreover, results obtained by the 2D model are more sensitive both to the bias conditions and to the change of device parameters. Interestingly, a look-like second harmonic oscillation has been observed at DC bias. We contribute the origin of divergences in simulation results to the different coupling path of electric field in the two models. And the second-harmonic oscillations at DC bias should be the result of the appearance of concomitant oscillations beside the channel excited by strong electric-field effects.
\end{abstract}

\section{Introduction}

Continuous miniaturization of semiconductor devices has led to high-speed operations and large-scale integrations of electronics in the past decades. A single silicon chip is now able to contain more than a billion transistors and operate at frequencies higher than $\mathrm{GHz}$. Benefitting from the utilization of planar architecture the operation frequency can be further increased. In planar devices, electrodes are connected side by side to the active semiconductor layer rather than being placed on top of each other, as in conventional multilayered vertical-structured devices. As a result, very low parasitic capacitances are obtainable, leading to a very high operating speed. Planar nanodevices based on 2DEGs in semiconductor heterostructures have been demonstrated to operate at tens of $\mathrm{GHz}$ or above [1-4]. In particular, self-switching diodes (SSDs) have been shown to possess zero threshold voltages and be able to detect electromagnetic signals with speed up to $110 \mathrm{GHz}$ and up to $2.5 \mathrm{THz}$ at room temperature and at a temperature of $150 \mathrm{~K}$, respectively $[3,5]$.

Apart from the experiments on planar nanodevices, there have been theoretical efforts based on analytical models or advanced numerical models, such as MC models, to further understand the device operations [6-8]. In MC models, electrons transporting in 2DEG layer is usually depicted by 2D EMC methods, and the electric field distributions are obtain by self-consistently solving 2D Poisson equations. This entirely $2 \mathrm{D}$ model not only obtains good agreements with experiments, but also predicts additional features of the planar devices, such as very low noise spectra in the $\mathrm{THz}$ range and enhanced $\mathrm{THz}$ detections by plasma [9-11]. However, the entirely 2D EMC models are unable to directly deal with electric field coupling beyond the 2DEG layer. To overcome this, a 2D-3D combined EMC model and a fully 3D EMC model have been developed, respectively. The previous model has been used to study the effects of electric 
field coupling through the substrate and the nearby dielectric layers on the performances of a side-gated transistor [12]. The later model has been applied to study three-terminal T-branch junctions (TBJs) with a top gate terminal, which is modeled as a side gate in entirely $2 \mathrm{D}$ models [13-15]. It is noteworthy that in spite of minimizing the need for parameter fitting and including the effect of electron transfer from channel to other layers, the 3D model leads to almost the same results as those obtained from 2D model for GaAs based devices [16]. However, in order to overcome huge time consuming, the EMC domain of 3D simulation should be limited to a small region.

In this work, efforts are devoted to study the influence of electric field coupling model on the simulation results of GaN based planar nanodevices. Unlike the GaAs based devices, the GaN based devices have much higher working voltage. This should lead to higher sensitivity of the device performances to the electric field coupling in the devices. As such, an entirely 2D EMC model and a 2D-3D combined EMC model are applied here and more attention will be paid to the phenomenon of Gunn oscillations that occur under high-field condition. The particular devices we focus on are SSDs, since they are not only based on electric field coupling but benefit for the propagation of Gunn domains [17]. The paper is structured as follows. In Section 2, the structure and working principle of the SSDs are firstly introduced and then the entirely 2D EMC model and the 2D-3D combined EMC model are concisely described, respectively. In Section 3, the performances of SSDs with different parameters are studied by both models under various conditions and results are analyzed in detail. In Section 4, the conclusions of this work are summarized.

\section{Device Working Principle and Numerical Models}

Figures 1(a) and 1(b) show schematically the top view and cross-section of a SSD. The device is based on a GaN/AlGaN heterostructure, where a $2 \mathrm{DEG}$ is formed at the GaN-AlGaN heterointerface with a carrier concentration of $8.0 \times 10^{12} \mathrm{~cm}^{-2}$ [18]. The two L-shaped insulating trenches are created by etching, through the 2DEG layer, ensuring that electrons have to pass the narrow channel between the two trenches in order to conduct a current between the left and right terminals. When a positive voltage is applied to the right terminal, positive charge is induced around the trenches, because of field effect, attracting electrons into the channel for the current to flow easily. However, when a negative voltage is applied to the right terminal, the negative charge around the trenches results in depletion of the channel, thus inhibiting the current flow, forming diode-like characteristics, as demonstrated by Song et al. [19].

The entirely $2 \mathrm{D}$ model is based on a semiclassical EMC method self-consistently coupled with Poisson equations [20]. As in earlier work, some theoretical assumptions are made in order to enhance the efficiency of the EMC simulations [11, 21, 22]. Firstly, 2D EMC simulations are performed only on the active GaN layer, since it is the $2 \mathrm{DEG}$ layer that

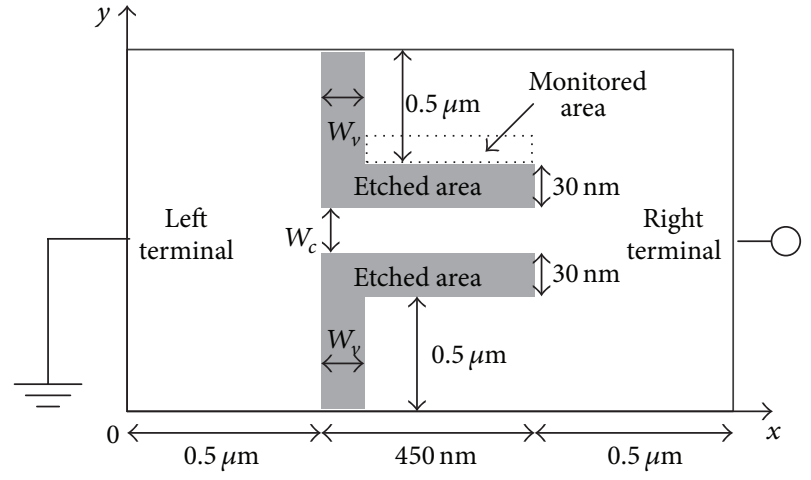

(a)

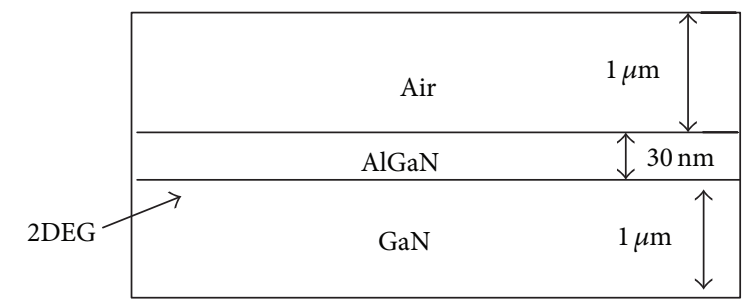

(b)

FIGURE 1: Schematic top view (a) and side view (b) of the simulated SSD (not to scale). The gray areas and the white area in the top view represent insulating trenches and $2 \mathrm{DEG}$, respectively. An interface of GaN/AlGaN heterostructures in (b) is just $30 \mathrm{~nm}$ below the device surface, at which a sheet of $2 \mathrm{DEG}$ forms. During simulations, all the insulating trenches are assumed to have vertical sidewalls and pass through both the entire AlGaN layer and the entire GaN one.

largely determines the electronic properties of the device. Secondly, to account for the fixed positive charges of the doping layer, a virtual net background doping $N_{V}=8.0 \times$ $10^{17} \mathrm{~cm}^{-3}$ (without ionized impurity scattering) is assigned to the GaN layer. In order to model the influence of surface states at semiconductor-air interface a uniform negative charge density, $N_{S}=0.8 \times 10^{12} \mathrm{~cm}^{-2}$, is also added at the edge of the insulating trenches during the simulations. Since the charge density is constant during the whole simulation process, this treatment is called constant charge (CC) model [18]. The SSD was designed with a channel length of $L_{c}=450 \mathrm{~nm}$ and a horizontal trench width of $W_{h}=30 \mathrm{~nm}$. Other geometric parameters are defined in Figure 1(a). All simulations are carried out at room temperature, with the left terminal grounded. And the Poisson equations are solved in $5 \mathrm{~nm} \times$ $5 \mathrm{~nm}$ meshes with a time step of $1 \mathrm{fs}$. More information about the model can be found in [21].

The 2D-3D combined EMC model is developed from the previous entirely $2 \mathrm{D}$ model. The $2 \mathrm{D}-3 \mathrm{D}$ model is also based on a semi-classical 2D EMC method but self-consistently coupled with 3D Poisson equations. In this model, we assume that electrons are all confined within the 2DEG layer, ignoring the effect of electron transfer from channel to other layers, so that 2D EMC method is enough to depict the micro behaviors of the electrons in the devices. When solving the Poisson equations, the charge related to the $2 \mathrm{DEG}$ is considered as 
a surface charge involved in a Neumann boundary condition. It has been shown in [12] that the 3D electric field coupling occurs in an effective distance of about $400 \mathrm{~nm}$ away from the 2DEG. As the distance between 2DEG and the device surface (top of the AlGaN layer) is only $30 \mathrm{~nm}$, the Poisson equations should be solved in a domain beyond the geometry of the devices. As shown in Figure 1(b), the GaN substrate and a region (Air) above the device surface, both with height of $1 \mu \mathrm{m}$, are included. In principle, the insulating trenches of the devices are only required to pass through the 2DEG. However, previous study showed that when the trench depth was less than $400 \mathrm{~nm}$, the change of the trench depth had nonnegligible influences on the device performances [12]. To avoid the influences coming from the variations of trench depth, all the trenches are assumed to have vertical sidewalls and pass through the whole GaN/AlGaN heterostructures from the device surface to the bottom of the substrate, which is with depth of $1030 \mathrm{~nm}$. Since the transfers of electrons along the $z$ direction are ignored, there should be no physical restrictions on the size of meshes [21]. As such and for reducing the computational effort, nonuniform meshes are utilized in the $z$ direction. Beginning from the 2DEG, with initial value of $1 \mathrm{~nm}$, both to the top of the simulated Air and to the bottom of the simulated substrate, the increase of mesh size obeys a special geometric progression. The common ratio used for the geometric progression changes from 2 to 1.5 and then to 1 when the size of the meshes is larger than $10 \mathrm{~nm}$ and $100 \mathrm{~nm}$, respectively. Dielectric constant used in the simulations for Air, AlGaN, and GaN is 1, 8.5, and 8.9, respectively. Other treatments are the same as the entirely $2 \mathrm{D}$ EMC model. Details of the above 2D-3D combined model can be found in our recent work [12].

\section{Simulation Results and Analysis}

Figure 2 shows the time-dependent output current of the SSD shown in Figure 1. During simulations, we set channel width of $W_{c}=30 \mathrm{~nm}$ and vertical trench width of $W_{v}=30 \mathrm{~nm}$, respectively. Other simulation parameters can be found in Figure 1. To further study this, we calculated the device current at different bias voltage steps, from 0 to $3.5 \mathrm{~V}$ at increments of $0.5 \mathrm{~V}$, for SSDs with different vertical trench widths and different channel widths. Then the right terminal of the SSD is biased to voltage steps from 4 to $24 \mathrm{~V}$ on an increase of $2 \mathrm{~V}$ for every $10 \mathrm{ps}$. Red solid line and green dashed line in Figure 2 represent simulation results obtained by the $2 \mathrm{D}$ model and the 2D-3D model, respectively. For both results, current peaks with sharp rise and fall can be found after each abrupt change of the applied voltage, which are anticipated as results of the charging of parasitic capacitances in the devices [17]. Apart from the above current peaks, simulation results obtained by both models are almost the same at bias lower than $4 \mathrm{~V}$. And when the bias is beyond $4 \mathrm{~V}$, output current obtained by the $2 \mathrm{D}$ model is higher than that obtained by 2D-3D model. However, the threshold voltages of Gunn oscillations are similar, $18 \mathrm{~V}$ by the $2 \mathrm{D}$ model and $16 \mathrm{~V}$ by the 2D-3D model. Since the length of the channel is $450 \mathrm{~nm}$, the threshold electric field for Gunn

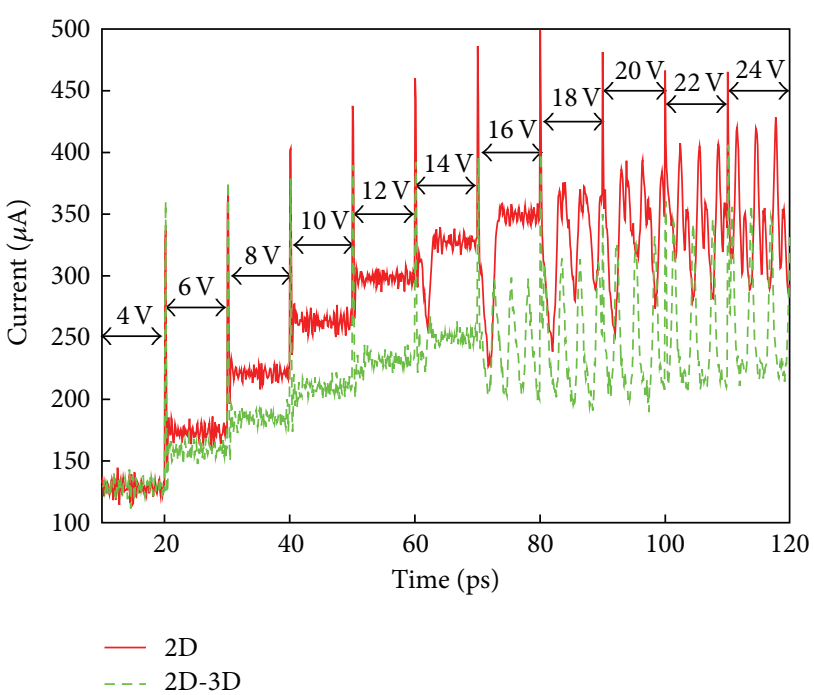

FIGURE 2: Output current of a SSD with channel width of $W_{c}=$ $30 \mathrm{~nm}$ and horizontal trench width of $W_{v}=30 \mathrm{~nm}$, when a series of time-dependent step voltages are applied at the right terminal. Red solid line is obtained using 2D model and green dashed line using 2D-3D combined model.

oscillations would be about $0.4 \mathrm{MV} / \mathrm{cm}$, which agrees with recent experimental results [23].

One can also find that the period of the oscillations at $18 \mathrm{~V}$ obtained by both models is about $3 \mathrm{ps}$, corresponding to a frequency of about $0.3 \mathrm{THz}$. However, the waveform of the oscillations obtained by the 2D-3D model is more sinusoidal than that by $2 \mathrm{D}$ model. This phenomenon becomes more obvious at higher bias, when a small additional peak emerges in every circle of oscillations obtained by the 2D model.

To understand the above bias-dependent divergence in simulation results, one should come back to the difference between the two models. As described in Section 2, in the 2D model, electric field couplings are well confined within the conducting layer (2DEG). However, in the 2D-3D model, part of electric field couples through the device substrate with longer path and through the Air with lower dielectric constant. As such, electric field couplings in the 2D-3D model are lower than those in the 2D model. Since SSDs are based on electric field effect $[2,8,21]$, it is reasonable that the device performances obtained by the $2 \mathrm{D}$ model are more sensitive to the bias conditions. Moreover, unlike the electric field couplings within the device, those through the Air are seldom affected by the device structure, so that results obtained by 2D-3D model would be less sensitive to the change of device geometry. To confirm this, SSDs with different geometrical parameters should be further studied by both models.

According to the above simulation results, the two models present large differences at high bias especially when Gunn oscillation occurs. As such, we focus on studying the waveforms of oscillations at bias of $26 \mathrm{~V}$. Shown in Figure 3 are the results for SSDs with different vertical trench width of $W_{v}=30 \mathrm{~nm}$ (red curves), $50 \mathrm{~nm}$ (green curves), and $70 \mathrm{~nm}$ (blue curves) obtained by (a) the 2D model and (b) the 2D-3D model. Other needed parameters are the same as those used 


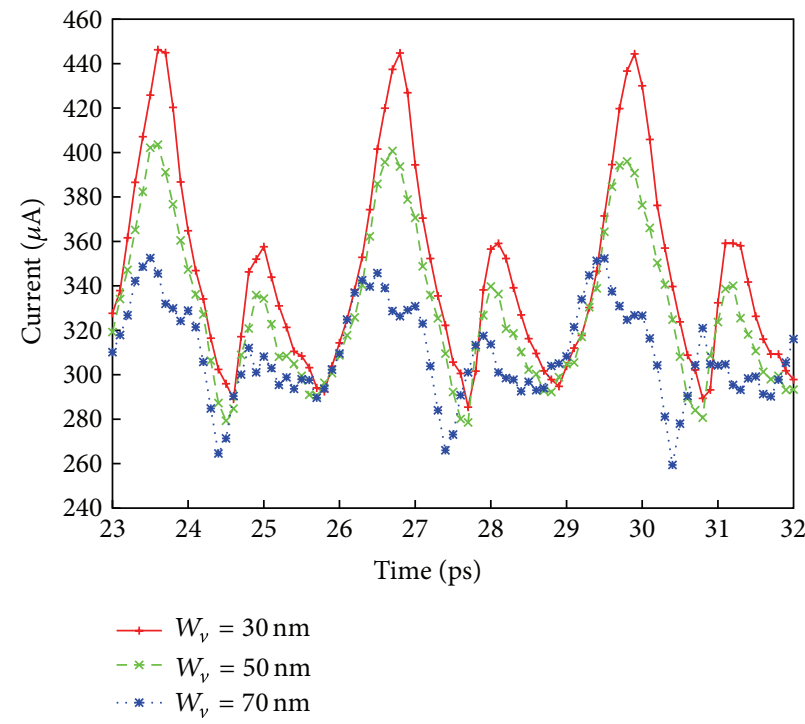

(a) $2 \mathrm{D}$

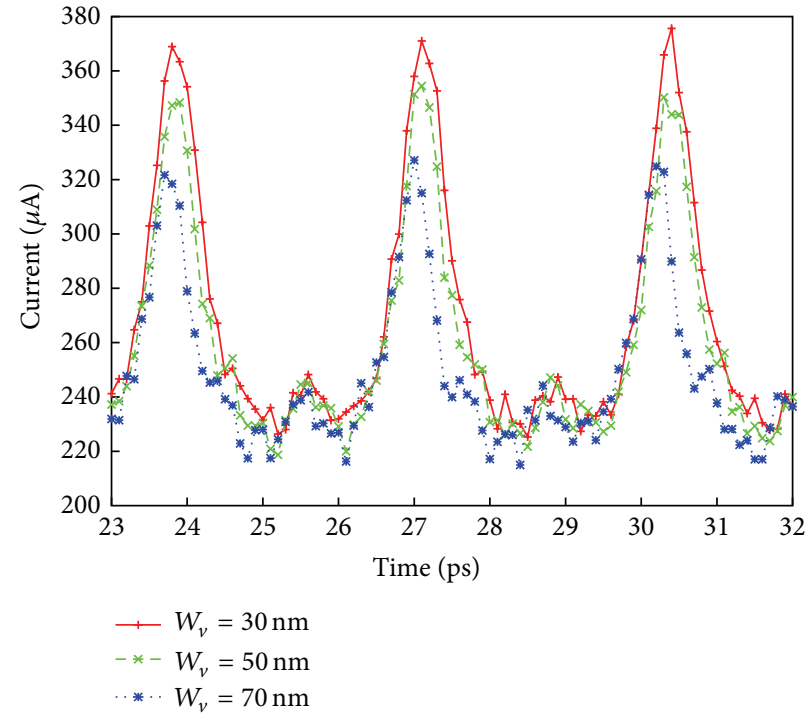

(b) $2 \mathrm{D}-3 \mathrm{D}$

FIGURE 3: Waveforms of oscillations of SSDs with different vertical trench width of $W_{v}=30 \mathrm{~nm}$ (red curves), $50 \mathrm{~nm}$ (green curves), and $70 \mathrm{~nm}$ (blue curves) at bias of $26 \mathrm{~V}$ obtained by (a) $2 \mathrm{D}$ model and (b) 2D-3D combined model (other simulation parameters are the same as those used in Figure 2).

in Figure 2. Comparing the results shown in Figure 3, one can find that the waveforms obtained by both models for narrow vertical trench, such as $W_{v}=30 \mathrm{~nm}$, show similar harmonic components, both with a small additional peak between two large major peaks. However, the amplitude of the additional peak obtained by the $2 \mathrm{D}-3 \mathrm{D}$ model is much lower than that obtained by the $2 \mathrm{D}$ model. Moreover, when the vertical trench width increases, the waveforms obtained by the $2 \mathrm{D}$ $3 \mathrm{D}$ model show just a small reduction in the amplitude. This reduction of the oscillation current is understandable. Since the vertical trench is widened, field effects applied on the channel are weakened [19]. As a result, the resistance of the channel increases, then the output current of the device reduces. In contrast, the waveforms obtained by the $2 \mathrm{D}$ model show not only a larger reduction in the amplitude, but also a nonnegligible change in the harmonic components, more additional peaks emerging.

In order to further reveal the divergences of simulation results obtained using different models, the influences of channel width on the waveforms of the oscillations have also been studied. Results are shown in Figure 4(a) using the 2D model and Figure 4(b) using the 2D-3D model. Simulation parameters are the same as those used in Figure 2, except that different channel widths with value of $50 \mathrm{~nm}$ (red curves), $60 \mathrm{~nm}$ (green curves), and $70 \mathrm{~nm}$ (blue curves) are chosen. One can find that additional peaks are also observed in Figure 4, which grow up with the increment of channel width. Once again, results obtained using the 2D model are more sensitive to channel width than those obtained using $2 \mathrm{D}-3 \mathrm{D}$ model. When the channel width is $70 \mathrm{~nm}$, the additional peak obtained by the $2 \mathrm{D}-3 \mathrm{D}$ model is only as high as $30 \%$ of the major peak, while that obtained by the $2 \mathrm{D}$ model is almost as high as the major peak. Since the additional peak happens to be in the middle of two nearby major peaks, the waveform looks like second harmonic (blue curve in Figure 4(a)) with frequency of about $0.6 \mathrm{THz}$.

It is noteworthy that similar phenomenon of second harmonic oscillations at DC bias was also observed in GaN based SSDs using an entirely 2D model [18]. In that work, based on a CC model to include the influence of surface states at semiconductor-air interface, the frequency of Gunn oscillations at DC bias had been proved to lie within the second generation band of oscillations taking place in the presence of an additional AC bias. Furthermore, a self-consistent charge (SCC) model proposed in [24] was also used to make a deeper study of the influences of surface charges on the performances of SSDs. Results obtained using such advanced charge model showed good agreements with experimental data under any bias condition. In the SCC model, the local value of the surface charge was dynamically adjusted depending on the surrounding carrier density resulting in an increase of surface charge density with bias, which suppressed Gunn oscillations in narrow channel devices. Similar suppressions of Gunn oscillations are also observed in our simulations when higher surface charge density is used (results not shown). Moreover, for wide channel devices results obtained based on SCC model exhibited qualitative dependencies and basic operation, including second harmonic oscillations at DC bias, as those obtained using CC model. For the authors who focused on the AC features of the SSDs, little attention was paid to understand the origin of second harmonic oscillations at DC bias.

It is known that for SSDs electrons surrounding the channel play an important role in the properties of electron transport in the channel. This is because the horizontal trenches used to define the channel are on the scale of 


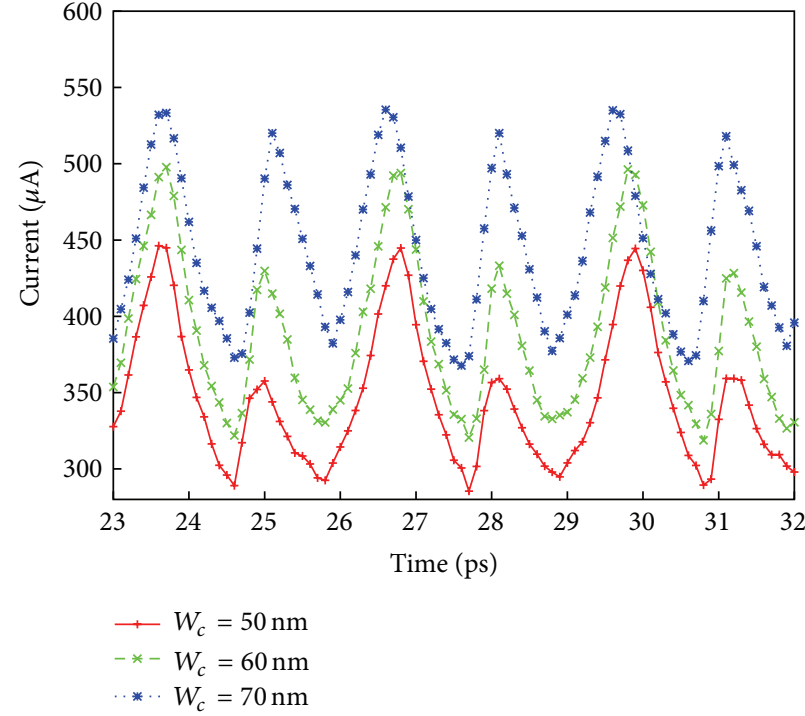

(a) $2 \mathrm{D}$

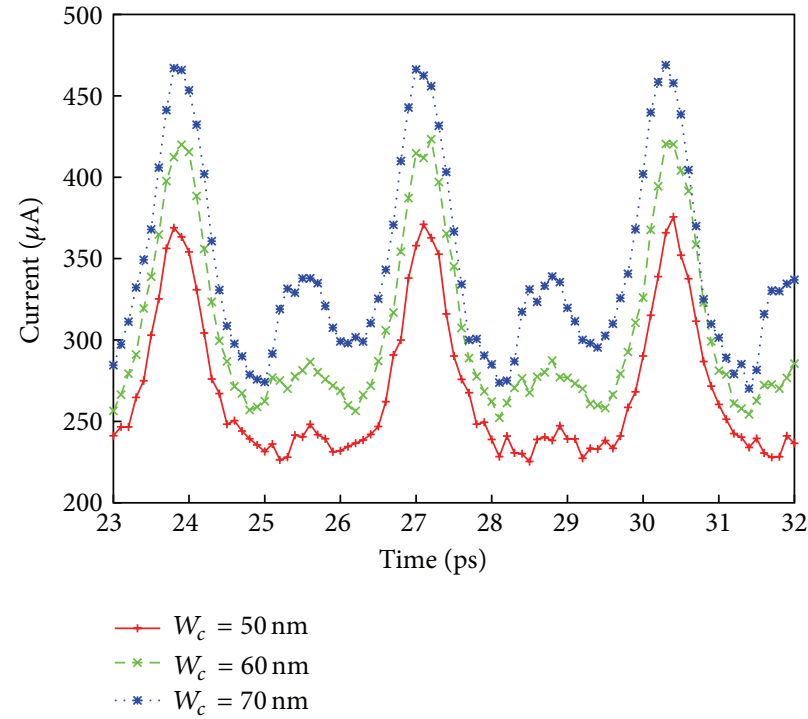

(b) $2 \mathrm{D}-3 \mathrm{D}$

FIGURE 4: Waveforms of oscillations in the SSDs with different channel width of $W_{c}=50 \mathrm{~nm}$ (red curves), $60 \mathrm{~nm}$ (green curves), and $70 \mathrm{~nm}$ (blue curves) at bias of $26 \mathrm{~V}$ obtained by (a) the $2 \mathrm{D}$ model and (b) the 2D-3D model (other simulation parameters are the same as those used in Figure 2).

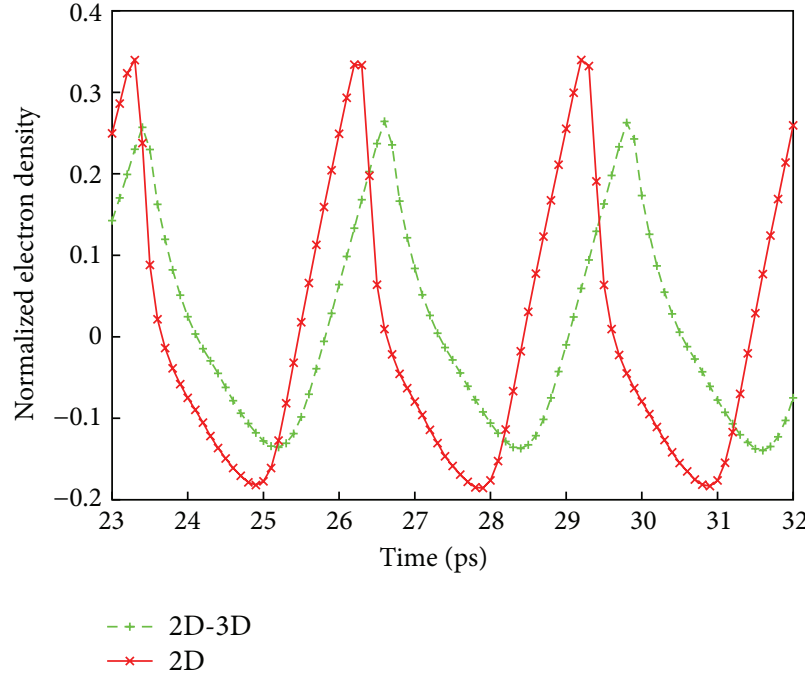

(a) Density fluctuation in channel

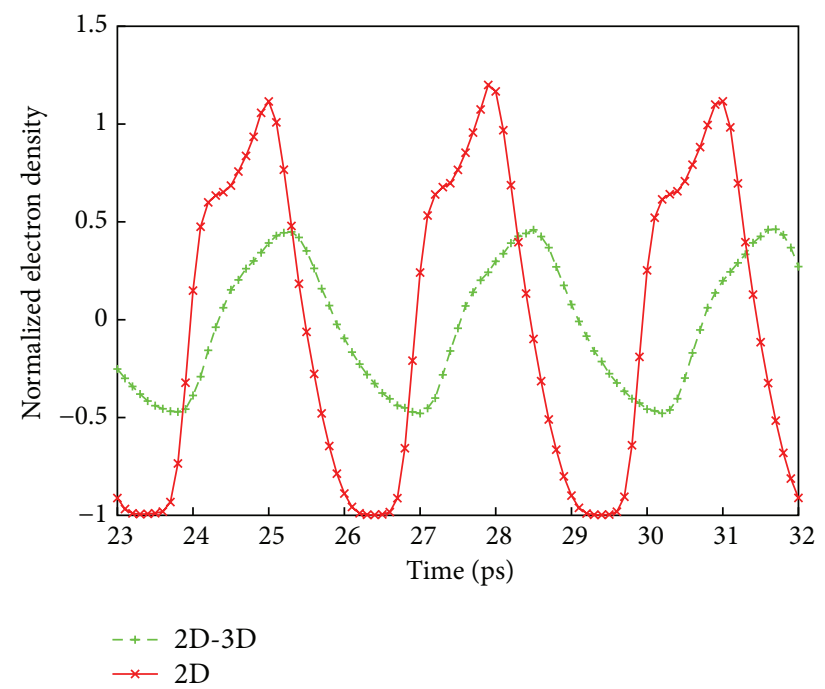

(b) Density fluctuation near channel

Figure 5: Normalized electron density fluctuations with time (a) in the channel and (b) near the channel (i.e., in the monitored area shown in Figure 1(a)) at bias of $26 \mathrm{~V}$ obtained by the 2D model (red curves) and the 2D-3D model (green curves). Other simulation parameters are the same as those used for obtaining blue curves in Figure 4.

nanometers resulting in strong electric field couplings of electrons on both sides of the trenches. As such, electron density fluctuations with time in the channel and in the monitored area indicated in Figure 1(a) have been studied for further understanding the device performances, especially the origin of second harmonic oscillations at DC bias. The monitored area is a rectangle with length and width of $420 \mathrm{~nm}$ and $35 \mathrm{~nm}$, respectively. Results are shown in Figure 5(a) in the channel and Figure 5(b) near the channel (i.e., in the monitored area). Red curves and green curves are obtained by the 2D model and the 2D-3D model, respectively. For convenience, results are normalized to time-averaged value. One can find that the changes of electron density with time in the channel obtained by both models are similar, showing obvious oscillations with the frequency about $0.3 \mathrm{THz}$. Such frequency is just the same as the fundamental frequency of the current oscillations, strongly implying that Gunn oscillations in the channel contribute to the appearance of the major peaks (or fundamental components of the current oscillations) shown in Figure 4. It is interesting 
that electron densities near the channel (in the monitored area) also show oscillations with frequency about $0.3 \mathrm{THz}$. These electron density oscillations are expected to impose an additional AC electric field on the channel, which should give rise to an increase of second harmonic component of the oscillations in the channel. At the same time, oscillations near the channel are just out of phase with those oscillations in the channel, resulting in a counteraction of the fundamental component of oscillations. These two mechanisms should be responsible for the origin of the second harmonic oscillations at DC bias observed in the simulations. Since DC bias only leads to constant distributions of electron potential around the channel, the above electron density oscillation near the channel should only be excited by electric field induced by the Gunn domains in the channel. Moreover, the amplitude of electron density oscillations near the channel obtained by the $2 \mathrm{D}-3 \mathrm{D}$ model is only half of that obtained by the $2 \mathrm{D}$ model. Recalling that the additional current peaks (shown in Figure 4) obtained by the 2D-3D model are also lower than those obtained by the 2D model, the understanding of the second harmonic oscillations at DC bias as a result of an accompanying of an oscillation near the channel can be further confirmed.

\section{Conclusion}

In this paper, we have carried out both entirely 2D EMC model and 2D-3D combined EMC model to analyze the performances of GaN based SSDs under different conditions. We find that both models give almost the same output current at bias lower than $4 \mathrm{~V}$. However, the output current obtained by the $2 \mathrm{D}$ model shows a larger increment at bias higher than $4 \mathrm{~V}$ and reaches oscillation state at bias of $18 \mathrm{~V}$ that is $2 \mathrm{~V}$ higher than that obtained by the $2 \mathrm{D}-3 \mathrm{D}$ model. We also find that current oscillations obtained by both models exhibit similar fundamental frequencies, but much different waveforms, especially at high bias. Moreover the deviation of waveform from sinusoidal wave with voltage and device parameters obtained by the $2 \mathrm{D}$ model is more distinguished than that obtained by the $2 \mathrm{D}-3 \mathrm{D}$ model. The above divergences in simulation results should come from the different coupling path of electric field in the two models. Interestingly, a look-like second-harmonic waveform has been obtained by the $2 \mathrm{D}$ model at DC bias. Further investigations show that electric field induced by Gunn domains in the channel has excited electron density oscillations outside the channel. It is these concomitant oscillations that contribute to the appearance of second harmonic oscillations at DC bias. Our studies also imply that for nanodevices with dominant electric field coupling and working at high bias, electric field distribution should be obtained by 3D Poisson equations; otherwise additional treatments should be introduced for an entirely 2D model.

\section{Conflict of Interests}

The authors declare that there is no conflict of interests regarding the publication of this paper.

\section{Acknowledgments}

This work was supported by FOK YING TONG Education Foundation (no. 122004), Natural Science Foundation of Guangdong Province, China (no. S2013010012711), and NSFC (nos. 11374185, 61072029, and U0934002). This work was also supported by the high-performance computing platform of South China Normal University. The authors thank anonymous reviewers for their constructive comments.

\section{References}

[1] A. M. Song, "Electron ratchet effect in semiconductor devices and artificial materials with broken centrosymmetry," Applied Physics A, vol. 75, no. 2, pp. 229-235, 2002.

[2] A. M. Song, P. Omling, L. Samuelson, W. Seifert, I. Shorubalko, and $\mathrm{H}$. Zirath, "Room-temperature and $50 \mathrm{GHz}$ operation of a functional nanomaterial," Applied Physics Letters, vol. 79, no. 9, pp. 1357-1359, 2001.

[3] C. Balocco, A. M. Song, M. Åberg et al., "Microwave detection at $110 \mathrm{GHz}$ by nanowires with broken symmetry," Nano Letters, vol. 5, no. 7, pp. 1423-1427, 2005.

[4] L. Worschech, F. Fischer, A. Forchel, M. Kamp, and H. Schweizer, "High frequency operation of nanoelectronic Ybranch at room temperature," Japanese Journal of Applied Physics, vol. 40, no. 8 B, pp. L867-L868, 2001.

[5] C. Balocco, M. Halsall, N. Q. Vinh, and A. M. Song, "THz operation of asymmetric-nanochannel devices," Journal of Physics Condensed Matter, vol. 20, no. 38, Article ID 384203, 2008.

[6] M. Åberg, J. Saijets, A. M. Song, and M. Prunnila, "Simulation and modeling of self-switching devices," Physica Scripta, vol. T114, p. 123, 2004.

[7] M. Åberg and J. Saijets, "DC and AC characteristics and modeling of Si SSD-nano devices," in Proceedings of the European Conference on Circuit Theory and Design, pp. 15-18, irl, September 2005.

[8] J. Mateos, B. G. Vasallo, D. Pardo, and T. González, "Operation and high-frequency performance of nanoscale unipolar rectifying diodes," Applied Physics Letters, vol. 86, no. 21, Article ID 212103, 3 pages, 2005.

[9] I. Iñiguez-de-la-Torre, J. Mateos, D. Pardo, and T. González, "Monte Carlo analysis of noise spectra in self-switching nanodiodes," Journal of Applied Physics, vol. 103, no. 2, Article ID 024502, 2008.

[10] I. Iñiguez-de-la-Torre, J. Mateos, D. Pardo, A. M. Song, and T. González, "Noise and terahertz rectification linked by geometry in planar asymmetric nanodiodes," Applied Physics Letters, vol. 94, no. 9, Article ID 093512, 2009.

[11] K. Y. Xu, X. F. Lu, A. M. Song, and G. Wang, "Terahertz harmonic generation using a planar nanoscale unipolar diode at zero bias," Applied Physics Letters, vol. 92, no. 16, Article ID 163503, 3 pages, 2008.

[12] K. Y. Xu, J. W. Xiong, A. M. Song, and G. Wang, "Effects of three-dimensional electric-field coupling on a side-gated nanotransistor," Semiconductor Science and Technology, vol. 26, no. 9, Article ID 095026, 6 pages, 2011.

[13] T. Sadi, F. Dessenne, and J.-L. Thobel, "Three-dimensional Monte Carlo study of three-terminal junctions based on InGaAs/InAlAs heterostructures," Journal of Applied Physics, vol. 105, no. 5, Article ID 053707, 2009. 
[14] T. Sadi and J.-L. Thobel, "Analysis of the high-frequency performance of InGaAs/InAlAs nanojunctions using a threedimensional Monte Carlo simulator," Journal of Applied Physics, vol. 106, no. 8, Article ID 083709, 2009.

[15] T. Sadi and J.-L. Thobel, "Study of the high-frequency performance of III-As nanojunctions using a three-dimensional ensemble Monte Carlo model," Journal of Physics: Conference Series, vol. 193, Article ID 012017, 2009.

[16] I. Iñiguez-de-la-Torre, T. González, D. Pardo et al., “Threeterminal junctions operating as mixers, frequency doublers and detectors: a broad-band frequency numerical and experimental study at room temperature," Semiconductor Science and Technology, vol. 25, no. 12, Article ID 125013, 2010.

[17] K. Y. Xu, G. Wang, and A. M. Song, "Gunn oscillations in a self-switching nanodiode," Applied Physics Letters, vol. 93, no. 23, Article ID 233506, 2008.

[18] A. Íñiguez-de-la-Torre, I. Íñiguez-de-la-Torre, J. Mateos et al., "Searching for THz Gunn oscillations in GaN planar nanodiodes," Journal of Applied Physics, vol. 111, no. 11, Article ID 113705, 2012.

[19] A. M. Song, M. Missous, P. Omling, A. R. Peaker, L. Samuelson, and W. Seifert, "Unidirectional electron flow in a nanometerscale semiconductor channel: a self-switching device," Applied Physics Letters, vol. 83, no. 9, pp. 1881-1883, 2003.

[20] C. Jacoboni and P. Lugli, The Monte Carlo Method for Semiconductor Device Simulation, Springer, New York, NY, USA, 1989.

[21] K. Y. Xu, X. F. Lu, G. Wang, and A. M. Song, "Strong spatial dependence of electron velocity, density, and intervalley scattering in an asymmetric nanodevice in the nonlinear transport regime," IEEE Transactions on Nanotechnology, vol. 7, no. 4, pp. 451-457, 2008.

[22] K. Y. Xu, X. F. Lu, A. M. Song, and G. Wang, "Enhanced terahertz detection by localized surface plasma oscillations in a nanoscale unipolar diode ," Journal of Applied Physics, vol. 103, Article ID 113708, 2008.

[23] N. Ma, B. Shen, F. J. Xu et al., "Current-controlled negative differential resistance effect induced by Gunn-type instability in n-type GaN epilayers," Applied Physics Letters, vol. 96, no. 24, Article ID 242104, 2010.

[24] I. Íñiguez-de-la-Torre, J. Mateos, T. González et al., "Influence of the surface charge on the operation of ballistic T-branch junctions: a self-consistent model for Monte Carlo simulations," Semiconductor Science and Technology, vol. 22, no. 6, pp. 663670, 2007. 

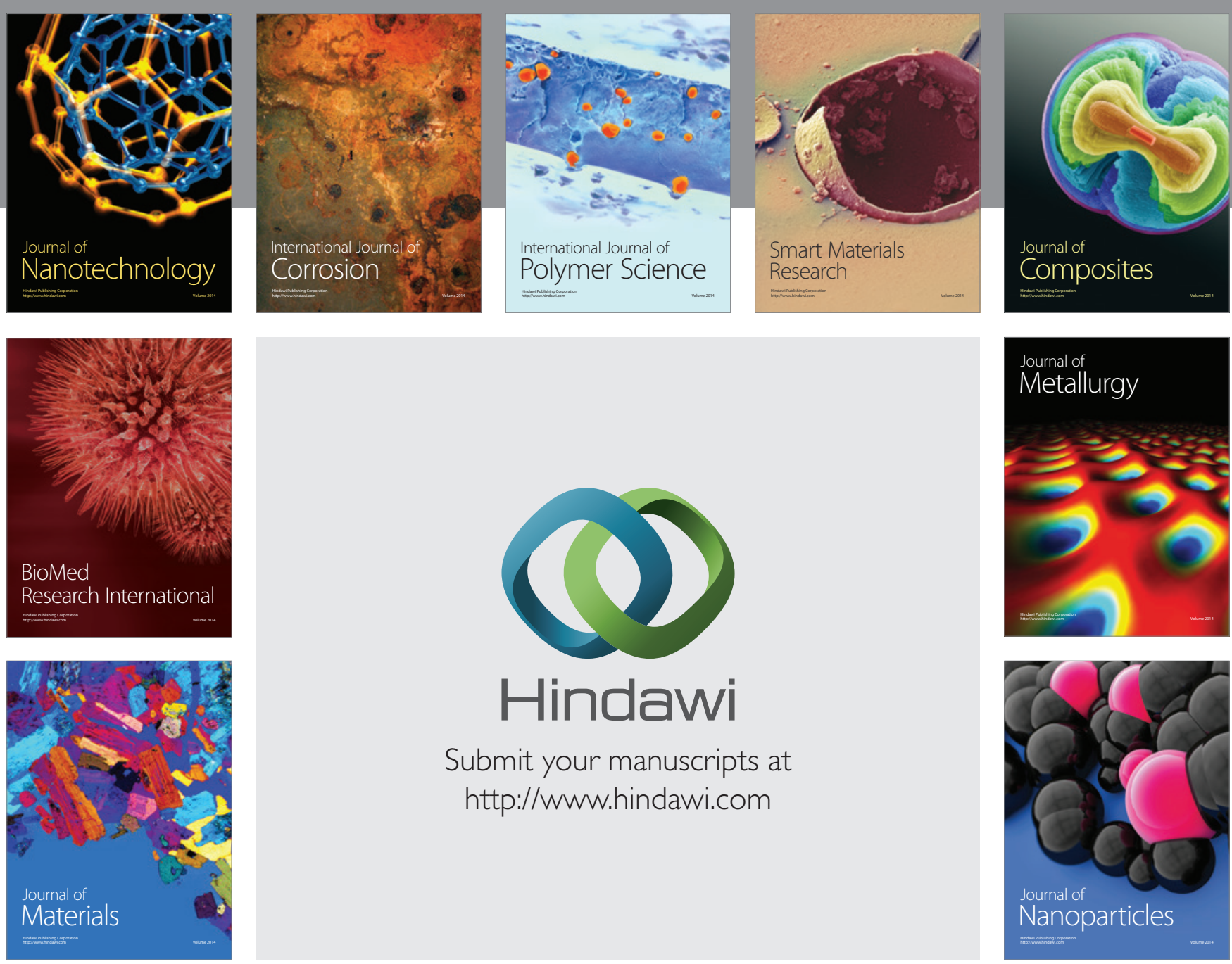

Submit your manuscripts at http://www.hindawi.com
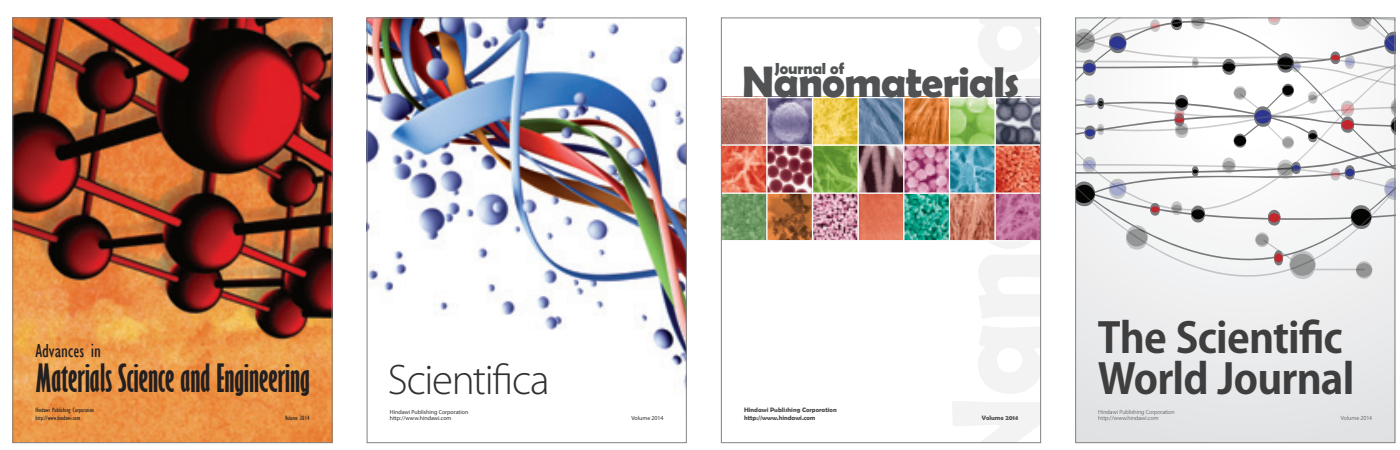

\section{The Scientific World Journal}
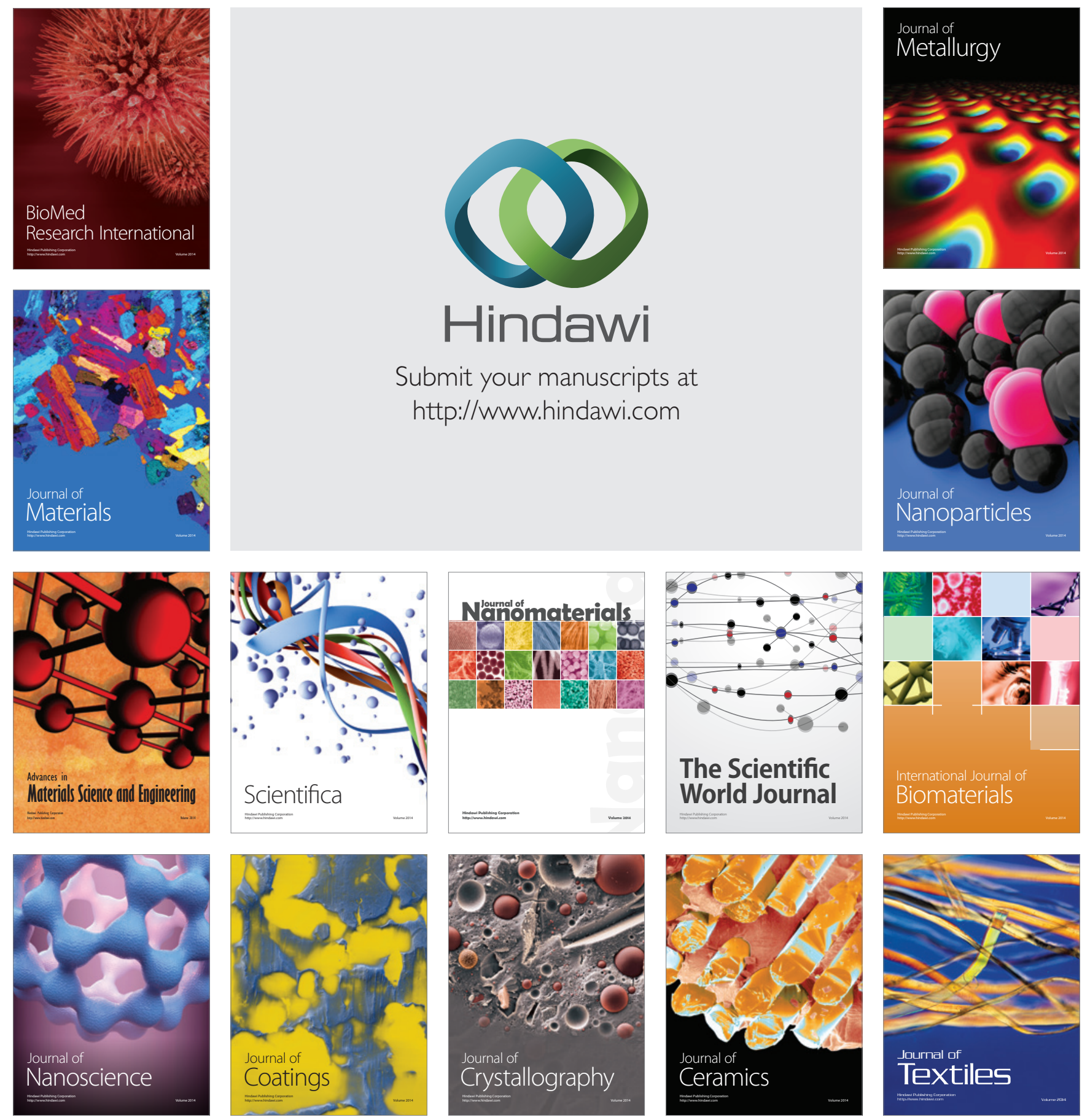